\title{
Respiratory and Blood Stream Infections are Associated with Subsequent Venous Thromboembolism After Primary Intracerebral Hemorrhage
}

\author{
Kara R. Melmed ${ }^{1,2^{*}}$, Amelia Boehme ${ }^{1,3}$, Natasha Ironside ${ }^{1}$, Santosh Murthy ${ }^{4}$, Soojin Park' , Sachin Agarwal ${ }^{1}$, \\ E. Sander Connolly ${ }^{5}$, Jan Claassen ${ }^{1}$, Mitchell S. V. Elkind ${ }^{1,3}$ and David Roh ${ }^{1}$
}

(๑) 2020 Springer Science+Business Media, LLC, part of Springer Nature and Neurocritical Care Society

\begin{abstract}
Background: Infection and venous thromboembolism (VTE) are associated with worse outcomes after intracerebral hemorrhage $(\mathrm{ICH})$. The relationship between infection and VTE in ICH patients is unclear. We hypothesized that infection would be associated with subsequent VTE after ICH.

Methods: We retrospectively studied consecutively admitted spontaneous primary ICH patients from 2009 to 2018 surviving beyond $24 \mathrm{~h}$. The primary predictor variable was infection, diagnosed prior to VTE. The primary outcome was VTE. We used multivariable logistic regression models to estimate the odds ratios and $95 \%$ confidence intervals $(\mathrm{OR}, 95 \% \mathrm{Cl})$ for $\mathrm{VTE}$ risk after infection of any type, after adjusting for $\mathrm{ICH}$ score, length of stay and days to deep venous thrombosis (DVT) prophylaxis. Similar analysis was done to estimate the association of infection subtypes, including respiratory and urinary and blood stream infections (BSI) with VTE.
\end{abstract}

Results: There were 414 patients (mean age 65 years, 47\% female) that met were analyzed. Infection was diagnosed in 181 (44\%) patients. Incident VTE was diagnosed in 36 (9\%) patients, largely comprised of DVT ( $n=32 ; 89 \%)$. Infection overall was associated with increased risk of subsequent VTE (adjusted OR 4.5, 95\% Cl 1.6-12.6). Respiratory (adjusted OR 5.7, 95\% Cl 2.8-11.7) and BSI (adjusted OR 4.0, 95\% Cl 1.3-11.0) were associated with future VTE. Urinary and other infections were not associated with subsequent VTE.

Conclusions: Infections are associated with subsequent risk of VTE among patients with $\mathrm{ICH}$. Further investigation is required to elucidate mechanisms behind this association and to improve VTE prevention after ICH.

Keywords: Intracranial hemorrhage, Venous thromboembolism, Infection, Complications, Inflammation, Thrombosis, Anticoagulants, Risk factors

\section{Introduction}

Infection and venous thromboembolic (VTE) events are independently associated with worse outcomes after intracerebral hemorrhage (ICH). The association

\footnotetext{
*Correspondence: kara.melmed@gmail.com

${ }^{1}$ Department of Neurology, Vagelos College of Physicians and Surgeons,

Columbia University, New York, NY, USA

Full list of author information is available at the end of the article
}

between infection and subsequent VTE in ICH patients is unclear. Patients with $\mathrm{ICH}$ are often immobile, placing them at increased risk for venous stasis and thrombotic events. Immobility is also a risk factor for some types of infection, such as pneumonia and decubitus ulcer infections. VTE occurs in 2-9\% of patients hospitalized with ICH [1-3], with an associated mortality of $26 \%$ when left untreated [3]. Less than $20 \%$ of patients with $\mathrm{ICH}$ are started on prophylactic anticoagulation (AC) [4] during 
their hospital stay, despite demonstration in meta-analyses of benefit from both initiation of VTE chemoprophylaxis [5] and resumption of therapeutic AC [6].

Infection is a known risk factor for thrombotic events in non ICH patients [7-9], possibly due to activation of the coagulation cascade by inflammatory mediators $[10,11]$. Infections occur in about $20-30 \%$ of patients admitted for $\mathrm{ICH}[12,13]$. Nosocomial infections in $\mathrm{ICH}$ patients are associated with hematoma volume, lower Glasgow Coma Scale (GCS), and deep location [11-13]. Infection in $\mathrm{ICH}$ patients is associated with greater discharge mortality and worse 90-day outcomes [11-13]. There has been some exploration into the association between infection and VTE occurrence in ICH patients [13]. The role of infection on timing of subsequent VTE risk during hospitalization for $\mathrm{ICH}$ patients has not been investigated. The relationship between type of infection and VTE occurrence is currently unknown in this population.

We hypothesized there would be an association between infection, infection subtypes, and subsequent VTE during ICH hospitalization. We further aimed to clarify the role of infection on timing of future VTE, as well as the relationship between infection type and VTE occurrence.

\section{Methods}

We performed a retrospective cohort study using data prospectively collected for $\mathrm{ICH}$ patients admitted to Columbia University Irving Medical Center as part of the ICH Outcomes Project. Consecutive primary ICH patients admitted between 2009 and 2018 were included. We excluded secondary etiologies of ICH (ICH secondary to underlying malignancy, vascular malformation, and hemorrhagic conversion following an ischemic stroke). Additionally, ICH patients with systemic coagulopathy (admission INR $>2.0$ or platelet count $<50 \times 10^{3}$ / $\mu \mathrm{L})$ as defined by SMASH-U criteria [14], prior VTE, or prior use of AC were excluded to best address infection exposure on incident VTE. Patients with early withdrawal of care or mortality within $24 \mathrm{~h}$ were excluded to avoid survival bias [7]. DVT chemoprophylaxis was started at the discretion of the attending physician in accordance with national guidelines [15] and institutional practice, typically $24-48 \mathrm{~h}$ from after evidence of hemorrhage stability proven by imaging.

\section{Measurements}

Baseline demographics, medical comorbidities, and clinical and radiographic ICH characteristics were recorded. ICH volume was obtained using semiautomatic hematoma size measurement software (Medical Imaging Processing, Analysis and Visualization software, National Institute of Health [NIH]). Severity scores including admission GCS, NIH Stroke Scale, and ICH score [16] were calculated at the time of admission for each patient by the admitting practitioner. Comorbid diseases and admission laboratories and data were also collected. Length of hospital stay (LOS) was calculated from NICU admission to hospital discharge.

Infection following ICH was our primary predictor variable and was defined as a composite event of blood stream infection (BSI), Clostridium difficile ( $C$. difficile), cellulitis or skin infection, pneumonia, urinary tract infection (UTI), or ventriculitis/meningitis. Hospital complications were recorded prospectively at the time of complication. These events were defined as per guideline criteria [17-21], and were further adjudicated in weekly meetings by a group of study physicians and coordinators. A diagnosis of BSI required culture positivity from sterile blood cultures. Gastrointestinal infection required the presence of diarrhea and PCR positivity for $C$. difficile [18]. Respiratory infections were defined as pneumonia in the setting of a combination of fever, leukocytosis, increased pulmonary secretions, increased oxygen requirements, focal consolidation on chest X-ray, and culture positive bronchial alveolar lavage when available [19]. UTI diagnosis was made in the setting of inflammatory urinalysis that reflexed to culture with positive bacteria [20]. Ventriculitis/meningitis diagnosis required cerebral spinal fluid with the combination of elevated white cell count, low glucose, elevated CSF lactate, and culture positivity [21]. The date of complication was documented as the date of collection of a positive culture or the date of clinical suspicion and initiation of antibiotics in the absence of culture data. Time to infection was calculated as NICU admission day to date of complication. Infections that occurred prior to incident $\mathrm{ICH}$ were not recorded. Patients who had the first infection diagnosed after their VTE event were categorized as having no infection prior to VTE.

The primary outcome was first VTE defined as deep venous thrombosis (DVT) and/or pulmonary embolism (PE). Diagnosis was made following clinical suspicion and confirmed with the appropriate imaging modality. Baseline VTE screening on admission did not occur. We did exclude patients with a history of VTE or hx of $\mathrm{AC}$ use to reduce likelihood of patients with underlying VTE prior to ICH event. Two independent reviewers reviewed charts for the presence of VTE as defined by positive imaging: Doppler ultrasound of extremity for DVT, and either CT angiogram of the chest or VQ scans for PE. Discrepancies were adjudicated by a third review. The date of VTE was documented by the date of confirmed DVT or PE by final imaging interpretation by 
an attending radiologist. Time to VTE was calculated as NICU admission day to date of complication.

\section{Statistical Methods}

Intergroup differences were explored via univariate analysis with Pearson's $\chi^{2}$ test for categorical variables and the Student's $t$ test for normally distributed continuous variables and Wilcoxon rank sum test when non-normally distributed. Adjusted logistic regression was performed to assess the association of infection to future VTE after adjusting for the following covariates chosen a priori: time to DVT prophylaxis, LOS, as well as ICH severity using ICH score. Logistic regression modeling was also used to study the relationship between infection type and thrombotic events, after adjusting for similar covariates. Significant intergroup differences thought to be potential confounders were also included into the model in sensitivity analyses. $p$ values were two-tailed and $p<0.05$ was considered significant. Analyses were performed using SPSS (version 25). We used the STROBE cohort checklist when writing our report [22].

\section{Results}

\section{Description of Cohort}

Of 738 patients admitted with ICH, 414 patients were included for analyses (Fig. 1). Of these, 181 (44\%) of patients carried a diagnosis of infection. Patients with infections during hospitalization presented with larger hematoma volumes and lower admission GCS (Table 1). There were more surgical evacuations of $\mathrm{ICH}$ in patients with infection. Hospital LOS was longer in patients who developed an infection. There were no other demographic differences between groups.

Incident VTE was diagnosed in $36(9 \%)$ patients, which were largely comprised of DVT $(n=32 ; 89 \%$ of VTE). There were no differences in median days [IQR] to DVT prophylaxis initiation in patients who went on to have a VTE (3[2-4]) versus those who did not $(3[2-3], p=0.1)$. Only seven (2\%) patients had VTE without preceding infection. Infection occurred without subsequent VTE in 153 (36\%) patients. Infection and VTE occurred together in $29(7 \%)$ patients. Of the total cohort, 225 (54\%) patients were free of both VTE and infectious complications. Only 54 (13\%) infections occurred prior to initiation of DVT prophylaxis. There was only one $(0.2 \%)$ VTE found prior to initiation of DVT prophylaxis. Median [IQR] days from infection to VTE was 12 [6-18].

\section{Association of Infection with VTE}

In the crude logistic regression model, infection was strongly associated with VTE (odds ratio [OR] 5.1, 95\% confidence interval (CI) 2.3-11.6; Table 2). Adjusting for disease severity, LOS, and days to DVT prophylaxis initiation, there continued to be an association with any infection and VTE (adjusted OR 4.5, 95\% CI 1.6-12.6).

\section{Association of Infection Subtype with VTE}

We did find a significant association between respiratory infection and VTE in crude logistic modeling (OR 5.7, 95\% CI 2.8-11.7) and after adjusting for disease severity, LOS, and days to DVT prophylaxis initiation (adjusted OR 5.7, 95\% CI 2.8-11.7) (Table 2). There was also an association between BSI and VTE in crude analysis (OR 6.9; 95\% CI 2.7-17.7) and after adjusting for disease severity, LOS, and days to DVT prophylaxis initiation (adjusted OR 4.0, 95\% CI 1.3-11.0). UTI, cellulitis, C. difficile, meningitis, and ventriculitis were not associated with future VTE.

\section{Sensitivity Analysis}

Given that there were significant intergroup differences in ICH volume and admission GCS, separate sensitivity analyses using these two covariates instead of ICH score were performed. Inclusion of ICH volume or admission GCS instead of ICH score did not change the association between any infection and future VTE (OR 4.4; 95\% CI 1.6-12.2). Additional sensitivity analyses including surgical evacuation as a covariate did not change the association of infection on subsequent VTE (OR 4.5; 95\% CI 1.6-12.5). Similarly, when performing sensitivity analyses adjusting for intergroup differences of lobar ICH location and female sex, we did not note a change in the association of infection and future VTE (OR 5.5; 95\% CI 1. 7-16.0).

\section{Discussion}

We identified an association of infection as well as specific infection subtypes with subsequent VTE after ICH. This is one of the largest cohorts used to examine the relationship between VTE and infection in ICH using prospective data collection. This is similar to findings from other patient populations [7] although most prior studies in $\mathrm{ICH}$ patients demonstrating risk factors for VTE did not analyze the effect of infection [3, 23-25]. Previous studies that did find associations between infection and VTE did not look at infection subtype, did not account for time between infection and VTE, and did not account for patients who developed VTE prior to infection $[1,11-13,26]$.

Longer LOS, sicker patients, lower GCS, and surgical interventions are all risk factors for infections. Subsequently, investigating the relationship between infection and VTE is complicated, as both disease severity [12] and LOS [13] are individually associated with both infection and VTE. We included LOS and markers of disease severity ( $\mathrm{ICH}$ score) as an adjustment variable in 


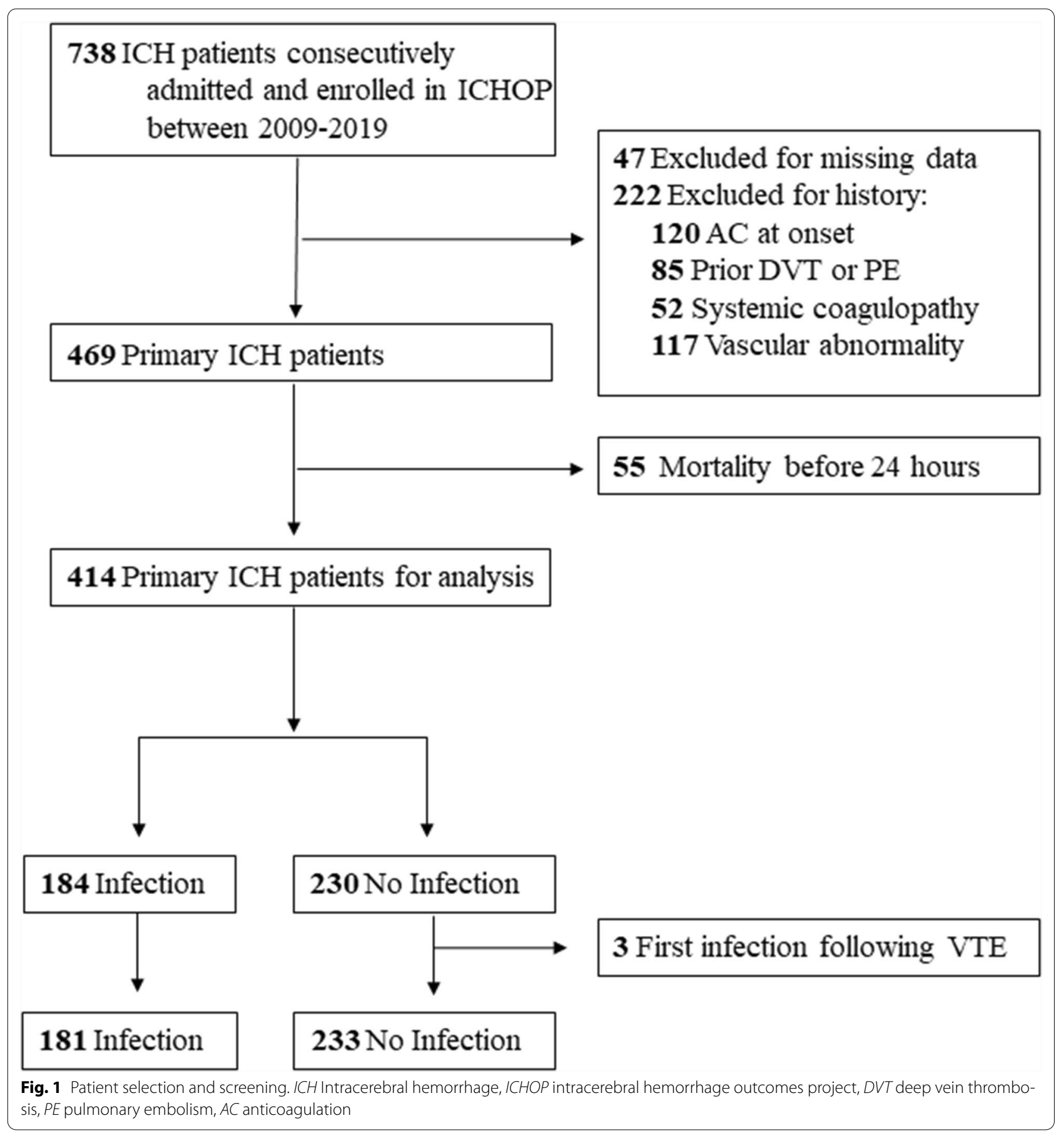

our regression model to account for these factors. It is worth noting that there were no differences in times to pharmacologic DVT prophylaxis between groups. And in parallel, the vast majority of patients who developed DVT encountered this complication outcome after the initiation of pharmacologic DVT prophylaxis. Our median [IQR] days to initiation of DVT prophylaxis was 3 [2-4] days and most likely reflects our center's practice of initiating DVT prophylaxis 24-48 h from hemorrhage stability on follow-up imaging. Assessment for hematoma expansion was beyond the scope of this study, but with longer times to image stability, one might hypothesize that these patients had a higher than expected time to stability. Further studies are in order to determine the biologic association between hematoma expansion and increased thrombotic risk. 
Table 1 Characteristics of patients, stratified by infection

\begin{tabular}{|c|c|c|c|c|}
\hline & Total $(N=414)$ & Infection $(N=181)$ & $\begin{array}{l}\text { No infection } \\
(N=233)\end{array}$ & $p$ value \\
\hline \multicolumn{5}{|l|}{ Demographics } \\
\hline Age, mean (SD) & $65(16)$ & $65(16)$ & $65(16)$ & 0.95 \\
\hline Female $^{\mathrm{a}}$ & $192(47)$ & $93(51)$ & $99(42)$ & 0.07 \\
\hline \multicolumn{5}{|l|}{ Ethnicity ${ }^{b}$} \\
\hline Caucasian & $107(26)$ & $53(30)$ & $54(23)$ & 0.15 \\
\hline \multicolumn{5}{|l|}{ Comorbidities } \\
\hline Atrial fibrillation/arrhythmia & $13(3)$ & $7(4)$ & $6(3)$ & 0.45 \\
\hline Diabetes & $101(25)$ & $50(28)$ & $51(23)$ & 0.2 \\
\hline Hypercholesteremia & $116(28)$ & $46(25)$ & $70(30)$ & 0.3 \\
\hline Hypertension & $332(80)$ & $144(79)$ & $188(81)$ & 0.8 \\
\hline \multicolumn{5}{|l|}{ Admission severity scores } \\
\hline GCS, median (IQR) & $12(7-15)$ & $9(7-13)$ & $14(9-15)$ & $<0.001$ \\
\hline Hematoma volume, median (IQR) & $13(4-31)$ & $19(7-39)$ & $8(3-23)$ & $<0.001$ \\
\hline ICH score, median (IQR) & $1(1-2)$ & $2(1-3)$ & $1(1-2)$ & $<0.05$ \\
\hline \multicolumn{5}{|l|}{$\mathrm{ICH}$ location ${ }^{c}$} \\
\hline Lobar & $178(50)$ & $74(45)$ & $104(55)$ & 0.06 \\
\hline Deep & $242(61)$ & $114(66)$ & $187(58)$ & 0.1 \\
\hline Infratentorial & $47(12)$ & $18(10)$ & $29(13)$ & 0.4 \\
\hline Admission temp, mean (SD) & $37(5)$ & $37(2)$ & $37(6)$ & 0.53 \\
\hline \multicolumn{5}{|l|}{ Admission labs } \\
\hline PTT, mean (SD) & $29(5)$ & $29(6)$ & $28(4)$ & 0.52 \\
\hline PT, mean (SD) & $13(2)$ & $13(2)$ & $13(2)$ & 0.45 \\
\hline Fibrinogen, mean (SD) & $523(187)$ & $542(193)$ & $487(173)$ & 0.33 \\
\hline Surgical evacuation & $44(10)$ & $29(16)$ & $15(6)$ & $<0.01$ \\
\hline Admission to infection, median days (IQR) & $4(2-7)$ & $4(2-7)$ & & \\
\hline $\begin{array}{l}\text { Time to first administration of DVT Prophylaxis, } \\
\text { median days (IQR) }\end{array}$ & $3(2-4)$ & $3(2-4)$ & $2(2-3)$ & $<0.001$ \\
\hline Hospital LOS, median days (IQR) & $9(5-21)$ & $19(9-31)$ & $6(4-11)$ & $<0.001$ \\
\hline
\end{tabular}

Bold values indicate significance

DVT Deep venous thrombosis, GCS Glasgow coma scale, ICH intracerebral hemorrhage, IQR interquartile range, LOS length of stay, $P T$ prothrombin time, $P T T$ partial thromboplastin time, $S D$ standard deviation

a Data are presented as number (\%) unless otherwise specified

b Self-reported by patients or their surrogates. Missing race/ethnicity data in $6 \%$ of patients

c Numbers do not sum to group totals because of overlap in location

While the longer LOS in more severe ICH patients likely contributes to increased opportunities for nosocomial infections, more severe ICH patients are also at increased risk for infection such as pneumonia in the setting of aspiration. While we attempted to control for admission clinical severity scores and LOS in our model to determine independent association between infection and subsequent DVT/PE event, it is possible that our findings may reflect the known relationship that more severe ICH injury has with greater risk for infection during hospitalization [12].

If there is indeed a relationship of infection with VTE independent of clinical severity and LOS, the mechanism for this association is uncertain. It could be a marker of underlying medical illness or a marker of the proinflammatory cascade leading to downstream endothelial dysfunction and thrombosis risk. It is known that both ischemic and hemorrhagic stroke induce local inflammation $[10,11,27]$, and it is possible that subsequent infection further triggers inflammatory responses, leading to excessive activation of coagulation $[9,28]$ and impairment of fibrinolysis [29] resulting in massive thrombin formation and fibrin deposition [8] and microvascular thrombosis. Infection, and notably sepsis, induces a marked pro-inflammatory and prothrombotic systemic environment [28]. These inflammatory 
Table 2 Infection and venous thromboembolism

\begin{tabular}{|c|c|c|c|c|c|}
\hline & $\begin{array}{l}\text { Total } \\
N=414(\%)\end{array}$ & VTE $(N=36)$ & No VTE $(N=378)$ & $\begin{array}{l}\text { Crude OR } \\
(95 \% \mathrm{CI})^{\mathrm{b}}\end{array}$ & $\begin{array}{l}\text { Adjusted OR } \\
(95 \% \mathrm{CI})^{c}\end{array}$ \\
\hline Any infection ${ }^{\mathrm{a}}$ & $181(44)$ & $28(78)$ & $153(41)$ & $5.1(2.3-11.6)$ & $4.5(1.6-12.6)$ \\
\hline Blood stream infection ${ }^{d}$ & $23(6)$ & $8(22)$ & $15(4)$ & $6.9(2.7-17.7)$ & $4.0(1.3-11.6)$ \\
\hline Pneumonia & $103(26)$ & $22(61)$ & $81(22)$ & $5.7(2.8-11.7)$ & $3.4(1.5-7.8)$ \\
\hline Urinary tract infection & $92(23)$ & $11(31)$ & $81(21)$ & $1.6(0.8-3.4)$ & $1.7(0.7-3.8)$ \\
\hline Other $^{\mathrm{e}}$ & $37(9)$ & $4(11)$ & $33(8)$ & $1.3(0.4-3.9)$ & $1.0(0.3-3.1)$ \\
\hline
\end{tabular}

Bold values indicate significance

CI Confidence interval, DVT deep venous thrombosis, ICH intracerebral hemorrhage, LOS length of stay, OR odds ratio, VTE venous thromboembolism

a Data are presented as number (\%) unless otherwise specified

b Univariate analysis, Pearson's Chi-square

c Multivariable logistic regression adjusting for ICH Score, LOS and days to first administration of DVT prophylaxis

d Numbers do not sum to group totals because of overlap in infection type

e Other; Clostridium Difficile, Cellulitis or Ventriculitis/Meningitis

mechanisms might explain the higher incidence of VTE in patients admitted with sepsis despite receiving guideline-recommended VTE prophylaxis [7].

Though our study strengths include prospectively collected data, consensus adjudication of complications (both infection and VTE), and a single-center cohort that uses a relatively homogenous diagnostic and treatment protocol for $\mathrm{ICH}$ and its complications, there are several notable limitations worth mentioning. The generalizability of the findings is limited given the single-center cohort and low numbers of VTEs in this study. The retrospective analysis created some limitation in accounting completely for comorbidities not initially collected. This study was also limited by the inclusion of only symptomatic VTE. Future studies that screen for both infection and VTE in all patients should be considered in order to identify and clarify the biologic link between infection and VTE. Additionally, the timing data regarding both infection and VTE onset were difficult to determine with certainty. Thus, the chronology between infection and VTE is somewhat speculative; however, the adjudication of hospital complications by group consensus helped mitigate this limitation. Furthermore, while the use of the date of diagnosis of infection and VTE rather than date of clinical suspicion may be seen as a limitation, using the latter would have most likely resulted in a shortened time to event and would most likely have strengthened the model. Further investigation is warranted to clarify the biologic drivers behind the association of infection and subsequent VTE as well as why particular infection types such as pulmonary and hematologic infection impact downstream VTE occurrence over others. Our findings could suggest the importance of adoption of early infection control practices not only in preventing infection, but in potentially preventing downstream VTE. However, further study into the impact on VTE utilizing specific techniques such as minimizing ventilator time with early weaning protocols, early Foley removal, minimizing use of CVCs, and early physical therapy including ambulation of patients even when ventilated would be informative for this cause. Conversely, further work is required to evaluate whether the development of infection necessitates not only the early initiation of pharmacologic DVT prophylaxis to prevent VTE in this high-risk patient population, but perhaps a more optimal dose. The inflammatory cascade set in motion in response to infection might be particularly damaging to this fragile population and perhaps earlier and more aggressive strategies for VTE prevention should be considered in ICH patients in the setting of infection.

\footnotetext{
Author details

${ }^{1}$ Department of Neurology, Vagelos College of Physicians and Surgeons, Columbia University, New York, NY, USA. ${ }^{2}$ Department of Neurology, NYU Langone Health, New York, NY, USA. ${ }^{3}$ Department of Epidemiology, Mailman School of Public Health, Columbia University, New York, NY, USA. ${ }^{4}$ Department of Neurology, Weill Cornell Medical Center, New York, NY, USA. ${ }^{5}$ Department of Neurosurgery, Vagelos College of Physicians and Surgeons, Columbia University, New York, NY, USA.
}

\section{Author Contributions}

KRM involved in conception and design, acquisition of data, analysis and interpretation of data, drafting the article, final approval of the version to be published. $A B$ involved in substantial contributions design, analysis and interpretation of data, revising the article critically for important intellectual content. NI, SM, SP, SA, ESC, and JC involved in acquisition of data, revising the article critically for important intellectual content. MSVE involved in substantial contributions to conception and design; revising the article critically for important intellectual content, final approval of the version to be published. DR involved in substantial contributions to conception and design, acquisition of data, revising it critically for important intellectual content, final approval of the version to be published.

\section{Sources of funding}

This research received no specific grant from any funding agency in the public, commercial, or not-for-profit sectors. Dr. Murthy reports grants from 
National Institutes of Health, grants from Leon Levy Foundation, grants from American Academy of Neurology Clinical Research Fellowship.

\section{Conflict of interest}

Dr. Claassen reports minority shares at iCE Neurosystems. The remaining authors declare that there is no conflict of interest.

\section{Ethical Approval/Informed Consent}

The study was approved by Columbia University Institutional Review Board. Consent for patients, or proxies when they were unable to consent for themselves, was completed at the time of presentation to the Neurosciences Intensive Care Unit (NICU).

\section{Publisher's Note}

Springer Nature remains neutral with regard to jurisdictional claims in published maps and institutional affiliations.

\section{Published online: 8 May 2020}

\section{References}

1. Kim KS, Brophy GM. Symptomatic venous thromboembolism: incidence and risk factors in patients with spontaneous or traumatic intracranial hemorrhage. Neurocrit Care. 2009;11:28-33. https://doi.org/10.1007/ s12028-009-9201-4.

2. Goldstein JN, Fazen LE, Wendell L, et al. Risk of thromboembolism following acute intracerebral hemorrhage. Neurocrit Care. 2009;10:28-34. https ://doi.org/10.1007/s12028-008-9134-3.

3. Stecker M, Michel K, Antaky K, et al. Risk Factors for DVT/PE in Patients with stroke and intracranial hemorrhage. Open Neurol J. 2014;8:1-6. https://doi.org/10.2174/1874205×01408010001.

4. Prabhakaran S, Herbers P, Khoury J, et al. Is prophylactic anticoagulation for deep venous thrombosis common practice after intracerebral hemorrhage? Stroke. 2015;46:369-75. https://doi.org/10.1161/strok eaha.114.008006.

5. Paciaroni M, Agnelli G, Venti M, et al. Efficacy and safety of anticoagulants in the prevention of venous thromboembolism in patients with acute cerebral hemorrhage: a meta-analysis of controlled studies. JThromb Haemost. 2011;9:893-8. https://doi.org/10.1111/j.1538-7836.2011.04241

6. Murthy SB, Gupta A, Merkler AE, et al. Restarting anticoagulant therapy after intracranial hemorrhage: a systematic review and meta-analysis. Stroke. 2017;48:1594-600. https://doi.org/10.1161/strokeaha.116.016327.

7. Kaplan D, Casper TC, Elliott CG, et al. VTE incidence and risk factors in patients with severe sepsis and septic shock. Chest. 2015;148:1224-30. https://doi.org/10.1378/chest.15-0287.

8. Semeraro F, Colucci M, Caironi P, et al. Platelet drop and fibrinolytic shutdown in patients with sepsis. Crit Care Med. 2018;46:e221-8. https://doi. org/10.1097/CCM.0000000000002919.

9. Semeraro N, Ammollo CT, Semeraro F, et al. Coagulopathy of acute sepsis. Semin Thromb Hemost. 2015:41:650-8. https://doi. org/10.1055/s-0035-1556730.

10. Dirnagl U, Klehmet J, Braun JS, et al. Stroke-induced immunodepression: experimental evidence and clinical relevance. Stroke. 2007;38:770-3. https://doi.org/10.1161/01.STR.0000251441.89665.bc

11. Boehme AK, Comeau ME, Langefeld CD, et al. Systemic inflammatory response syndrome, infection, and outcome in intracerebral hemorrhage.
Neurol Neuroimmunol Neuroinflamm. 2018;5:e428. https://doi org/10.1212/nxi.0000000000000428.

12. Lord AS, Langefeld CD, Sekar $P$, et al. Infection after intracerebral hemorrhage: risk factors and association with outcomes in the ethnic/racial variations of intracerebral hemorrhage study. Stroke. 2014;45:3535-42. https://doi.org/10.1161/strokeaha.114.006435.

13. Murthy SB, Moradiya Y, Shah J, et al. Nosocomial infections and outcomes after intracerebral hemorrhage: a population-based study. Neurocrit Care. 2016:25:178-84. https://doi.org/10.1007/s12028-016-0282-6.

14. Meretoja A, Strbian D, Putaala J, et al. SMASH-U: a proposal for etiologic classification of intracerebral hemorrhage. Stroke. 2012;43:2592-7. https //doi.org/10.1161/strokeaha.112.661603.

15. Hemphill JC 3rd, Greenberg SM, Anderson CS, et al. Guidelines for the management of spontaneous intracerebral hemorrhage: a guideline for healthcare professionals from the American Heart Association/American Stroke Association. Stroke. 2015:46:2032-60. https://doi.org/10.1161/ str.0000000000000069.

16. Hemphill JC 3rd, Bonovich DC, Besmertis L, et al. The ICH score: a simple, reliable grading scale for intracerebral hemorrhage. Stroke. 2001;32:891-7.

17. Bailey E, Kroshinsky D. Cellulitis: diagnosis and management. Dermatol Ther. 2011:24:229-39. https://doi.org/10.1111/j.1529-8019.2011.01398.x.

18. Napolitano LM, Edmiston CE Jr. Clostridium difficile disease: diagnosis, pathogenesis, and treatment update. Surgery. 2017;162:325-48. https:// doi.org/10.1016/j.surg.2017.01.018.

19. Erb CT, Patel B, Orr JE, et al. Management of adults with hospitalacquired and ventilator-associated pneumonia. Ann Am Thorac Soc. 2016;13:2258-60. https://doi.org/10.1513/annalsats.201608-641cme.

20. Rowe TA, Juthani-Mehta M. Diagnosis and management of urinary tract infection in older adults. Infect Dis Clin N Am. 2014;28:75-89. https://doi. org/10.1016/j.idc.2013.10.004.

21. Tunkel AR, Hasbun R, Bhimraj A, et al. Infectious diseases society of America's clinical practice guidelines for healthcare-associated ventriculitis and meningitis. Clin Infect Dis. 2017. https://doi.org/10.1093/cid/ciw861.

22. von Elm E, Altman DG, Egger $M$, et al. The strengthening the reporting of observational studies in epidemiology (STROBE) statement: guidelines for reporting observational studies. Lancet. 2007;370:1453-7. https://doi. org/10.1016/S0140-6736(07)61602-X.

23. Ding $D$, Sekar P, Moomaw CJ, et al. Venous thromboembolism in patients with spontaneous intracerebral hemorrhage: a multicenter study. Neurosurgery. 2018. https://doi.org/10.1093/neuros/nyy333.

24. Christensen MC, Dawson J, Vincent C. Risk of thromboembolic complications after intracerebral hemorrhage according to ethnicity. Adv Ther. 2008:25:831-41. https://doi.org/10.1007/s12325-008-0092-0.

25. Ogata T, Yasaka M, Wakugawa Y, et al. Deep venous thrombosis after acute intracerebral hemorrhage. J Neurol Sci. 2008;272:83-6. https://doi. org/10.1016/j.jns.2008.04.032.

26. Investigators CIT. Venous thromboembolism after intraventricular hemorrhage: results from the CLEAR III trial. Neurosurgery. 2018. https://doi. org/10.1093/neuros/nyy189.

27. Kamel $\mathrm{H}$, ladecola C. Brain-immune interactions and ischemic stroke: clinical implications. Arch Neurol. 2012;69:576-81. https://doi. org/10.1001/archneurol.2011.3590.

28. Levi M, van der Poll T. Coagulation and sepsis. Thromb Res. 2017;149:3844. https://doi.org/10.1016/j.thromres.2016.11.007.

29. Gando S. Role of fibrinolysis in sepsis. Semin Thromb Hemost. 2013;39:392-9. https://doi.org/10.1055/s-0033-1334140. 